\title{
Review of: "Activation of the CaMKII-Sarm1-ASK1 MAP kinase pathway protects against axon degeneration caused by loss of mitochondria"
}

\author{
Clara De Palma ${ }^{1}$ \\ 1 University of Milan
}

Potential competing interests: The author(s) declared that no potential competing interests exist.

The aim of this manuscript is to assess the molecular mechanism by which CaMKII can protect against axon degeneration due to the loss of axonal mitochondria

The manuscript contains a substantial amount of data to characterize the distinct events involved in this specific neuronal damage. The authors describe how CamKII activation suppresses axon degeneration requiring Sarm activity and NSY-SEK1-MAPK pathway and how its axonal localization depends on mitochondria.

They also investigate the role of calsyntenin in controlling CaMKII activity and neuronal degeneration, supporting a model in which disruption of CASY-1 trafficking complex prevents axon degeneration in absence of mitochondria, also involving L-type calcium channels and their localization.

A strength of the study is certainly that multiple aspects of CaMKII involvement in its anti-degenerative role are dissected by using several mutants and novel approaches, otherwise, this makes the manuscript too heavy and with too many figures. Overall, the quality of the manuscript and the science underneath it are high. However, the large number of experiments often confuses the reader distracting him from the importance and relevance of the addressed features.

The goal here should be to provide a better rationale for why certain experiments are included. It would be even more valuable if the authors could guide the reader in better understanding the biological meaning of their observations.

I am aware that this model offers a great opportunity for an unbiased screening, by contrast, it is very difficult to follow the experimental flow with all these mutants.

\section{Comments:}

In your model of mitochondrial depletion, axon protection is associated with a specific loss of axonal CaMKII while it is retained in the cell body. Why its activity in the cell body is not enough to protect from degeneration? Which is the role of CaMKII specifically in axons? How could the activation of a specific MAPK pathway prevent degeneration in absence of mitochondria? 
maintenance, and I am really puzzled about that. Moreover, I missed the point on how CASY-1 can mediate degeneration transporting LIN-10.

Along this line, I am also confused about the process directed correlated with mitochondria depletion: which are the events that could be directly restored by mitochondrial recovery?

Another remark is the possibility to translate these mechanisms in mammalian cells. Could you confirm your results in mammalian models of axons degeneration associated with defects in mitochondria localization or function?

Could you try to differently manipulate (not genetically) these axes to suppress degeneration? Could you select a different model of axon degeneration to assess whether these mechanisms are specifically associated with mitochondrial depletion? 